\title{
Determinação e modelagem das propriedades físicas e da contração volumétrica do trigo, durante a secagem
}

\author{
Paulo C. Corrêa ${ }^{1}$, Deise M. Ribeiro ${ }^{2}$, Osvaldo Resende ${ }^{3}$ \& Fernando M. Botelho ${ }^{4}$
}

\begin{abstract}
RESUMO
O presente trabalho foi realizado com o objetivo de se avaliar o efeito da variação do teor de água nas propriedades físicas dos grãos de trigo e ajustar diferentes modelos matemáticos aos valores experimentais, em função do teor de água do produto; além disso, analisaram-se duas metodologias de determinação da massa específica aparente e da massa específica real. Com base nos resultados obtidos, concluiu-se que a redução do teor de água influencia as propriedades físicas dos grãos de trigo, provocando a diminuição da porosidade, o aumento da massa específica aparente e da massa específica real. Outrossim, os valores das massas específicas real e aparente, calculados a partir da composição química dos grãos de trigo, são inferiores aos valores experimentais; contudo, as diferenças entre os valores obtidos pelas duas metodologias diminuem com a redução do teor de água do produto. O modelo de Bala \& Woods adaptado é o que melhor se ajusta aos dados experimentais de contração volumétrica do grão e da massa de grãos de trigo. Embora tenha ocorrido contração de volume dos grãos de trigo, sua forma (esfericidade e circularidade) não varia durante o processo de secagem, para a faixa de umidade estudada.
\end{abstract}

Palavras-chave: massa específica, porosidade, modelo matemático, encolhimento

\section{Determination and modeling of physical properties and shrinkage of wheat grains during the drying process}

\begin{abstract}
The objectives of this work was to evaluate the effect of variation of water content on the physical properties of wheat grain and to adjust different mathematical models to the obtained experimental values as a function of moisture content. Also two different methodologies for determining bulk density and the real density were analysed. Based on the obtained results it may be concluded that the reduction of the moisture content affects the physical properties of wheat grain promoting the decrease of porosity and increase of both the bulk density and the specific gravity. Also the wheat grain bulk density and specific and real gravity values found through the chemical composition of grain were lower than the experimental data. However, the differences in values found between the two methodologies decreased as the moisture content decreases. The adapted Bala \& Woods model fits best the individual wheat grain shrinkage as well as the bulk grain volume shrinkage. The grain shape (sphericity and circularity) did not vary for the moisture content range in this experiment.
\end{abstract}

Key words: density, porosity, mathematical model, shrinkage

1 DEA/UFV, CEP. 36571-000, Viçosa, MG. Fone: (31) 3899-2118. E-mail: copace@ufv.br

${ }^{2}$ Doutoranda em Engenharia Agrícola/UFV, Rua Professor Alberto Pacheco 202, apt 40, Bairro Ramos, CEP 36570-000, Viçosa, MG. E-mail: deise eng@yahoo.com.br

${ }^{3}$ Doutorando em Engenharia Agrícola/UFV. E-mail: oresende@vicosa.ufv.br

${ }^{4}$ Graduando em Engenharia Agrícola/UFV. E-mail: fernando.eaa@gmail.com 


\section{INTRODUÇÃO}

O trigo é um produto de origem asiática e apresenta ampla importância comercial, sendo utilizado como matériaprima para a fabricação de uma extensa gama de produtos alimentícios industrializados.

A produção de grãos sadios e de qualidade superior requer que o produto seja colhido antecipadamente, ou seja, quando ainda apresenta elevado teor de água, visando minimizar as perdas ocasionadas no campo pelo ataque de insetos e microrganismos.

A secagem dos produtos agrícolas é o processo mais utilizado para assegurar sua qualidade e estabilidade após a colheita diminuindo, assim, a quantidade de água do material e conseqüentemente a atividade biológica e as mudanças químicas e físicas que ocorrem durante o armazenamento; no entanto, o fenômeno de redução do teor de água de grãos envolve, simultaneamente, processos de transferência de calor e massa, que podem alterar, de forma substancial, a sua qualidade e as propriedades físicas, dependendo do método e das condições de secagem (Hall, 1980).

As propriedades físicas dos grãos são características relevantes na otimização dos processos industriais e no desenvolvimento de novos projetos e equipamentos utilizados nas operações pós-colheita. Variações dessas propriedades em função do teor de água e de outros fatores durante a secagem de vários produtos, têm sido investigadas por diversos autores (Mcminn \& Magee, 1997; Ruffato et al., 1999).

A massa específica aparente ou peso hectolitro $(\mathrm{PH})$ do trigo é uma propriedade que assume elevada importância para efeito de comercialização do produto, uma vez que os preços praticados consideram esta característica como um indicativo de qualidade e rendimento. No Brasil, o trigo limpo com teor de água de $13 \%$ b.u. é comercializado utilizandose, como referência, um valor de $\mathrm{PH}\left(\mathrm{kg} \mathrm{hL}^{-1}\right)$ igual a 78; assim, o conhecimento mais apurado do comportamento desta propriedade física durante a secagem é crucial para se obter um rendimento final e melhor preço do produto.

A massa específica real ou unitária de partículas sólidas granulares, como grãos e cereais, pode ser estimada, de acordo com Lewis (1993), a partir do conhecimento da composição química do produto. Afonso Júnior et al. (2000) empregaram esta metodologia para o cálculo da massa específica real em grãos de milheto utilizando os dados da massa específica dos seus componentes químicos e compararam os valores aos obtidos, em função da porosidade e da massa específica aparente. As diferenças entre os resultados encontrados foram inferiores a $6,0 \%$; deste modo, os autores sugerem que as duas metodologias apresentam boa estimativa dos valores da massa específica real dos grãos de milheto.

A representação matemática do processo de secagem de diversos produtos agrícolas vem, há algum tempo, sendo estudada e utilizada na tentativa de predizer os fenômenos que acontecem durante este processo.

A maioria dos modelos empregados para representar a secagem dos produtos agrícolas foi desenvolvida negligenciando-se a contração volumétrica do produto durante o pro- cesso de desidratação (Brooker et al., 1992). Referidas equações vêm sendo revisadas para incorporar o efeito desse fenômeno para uma simulação melhor do processo e precisão dos resultados (Lang \& Sokhansanj, 1993).

Devido à sua desidratação, as mudanças volumétricas dos produtos são relatadas como sendo as primeiras causas das alterações das principais propriedades físicas de produtos agrícolas (Ratti, 1994; Sokhansanj \& Lang, 1996).

Ratti (1994) e Zogzas et al. (1994) observaram que a contração volumétrica de produtos vegetais durante a secagem não é função exclusiva do teor de água mas, também, dependentes das condições do processo e da geometria do produto.

As bases teóricas para o conhecimento do processo de contração volumétrica envolvem complexas leis mecânicas e de deformação de materiais (Towner, 1987); no entanto, muitos pesquisadores utilizam aproximações e modelos empíricos, na tentativa de melhor representarem este complicado fenômeno em produtos de natureza biológica (Lang et al., 1994; Krokida \& Maroulis, 1997; Afonso Júnior et al., 2000; Mayor \& Sereno, 2004).

Considerando o exposto e a importância e variabilidade existentes nas propriedades físicas dos produtos agrícolas objetivou-se, com o presente trabalho:

- determinar as propriedades físicas dos grãos de trigo de uma variedade nacional, ao longo do processo de secagem

- analisar as diferentes metodologias de determinação da massa específica aparente e da massa específica real

- avaliar a contração volumétrica dos grãos de trigo durante a secagem

- ajustar diferentes modelos matemáticos aos valores experimentais das propriedades analisadas, em função do teor de água.

\section{MATERIAL E MÉTODOS}

Este trabalho foi desenvolvido no Laboratório de Propriedades Físicas e Qualidade de Produtos Agrícolas, pertencente ao CENTREINAR, localizado na Universidade Federal de Viçosa, Viçosa, MG; para sua realização, utilizaram-se grãos de trigo, cultivar Aliança, desenvolvida na UFV, colhidos com teor de água de aproximadamente 0,32 (b.s.).

A secagem foi realizada em estufa com ventilação forçada mantida a temperatura de $40{ }^{\circ} \mathrm{C}$; o acompanhamento da redução do teor de água ao longo do tempo de secagem, foi realizado pelo método gravimétrico (perda de massa), conhecendo-se o nível de umidade inicial do produto, até atingir o teor de água final de 0,10 (b.s.). Para este monitoramento foram empregados um cronômetro digital e uma balança analítica com precisão de $0,01 \mathrm{~g}$.

$\mathrm{O}$ teor de água do produto foi determinado pelo método da estufa, $105 \pm 3{ }^{\circ} \mathrm{C}$, durante $24 \mathrm{~h}$, com três repetições (Brasil, 1992); enfim, para cada teor de água obtido, as amostras foram homogeneizadas e encaminhadas para determinação das propriedades físicas e da contração volumétrica. 


\section{Porosidade}

A porosidade da massa de trigo foi definida pela média de três repetições, utilizando-se um picnômetro de comparação a ar, segundo o processo descrito por Day, apud Mohsenin (1986).

\section{Massa específica aparente e real}

A massa específica aparente $\left(\rho_{\mathrm{ap}}\right)$ foi mensurada através de uma balança de peso hectolitro, com capacidade de um litro, em três repetições para cada tratamento.

Obteve-se a massa específica real $(\rho)$ em função da porosidade $(\varepsilon)$ e da massa específica aparente $\left(\rho_{\mathrm{ap}}\right)$ de acordo com a equação descrita por Mohsenin (1986):

$$
\rho=\frac{\rho_{\text {ap }}}{(1-\varepsilon)}
$$

Os resultados experimentais das massas específicas aparente e real foram comparados aos obtidos pelo cálculo a partir da composição química média dos grãos de trigo, adaptando-se a metodologia descrita por Lewis (1993), de acordo com a equação abaixo:

$$
\rho=\frac{1+\mathrm{U}^{*}}{\left(\mathrm{U}^{*} / \rho_{\mathrm{ag}}\right)+\left(\omega_{1} / \rho_{1}\right)+\cdots+\left(\omega_{\mathrm{n}} / \rho_{\mathrm{n}}\right)}
$$

em que:

$\rho_{\mathrm{ag}}$ - massa específica da água, $1000 \mathrm{~kg} \mathrm{~m}^{-3}$

$\mathrm{U}^{*}$ - teor de água do produto, decimal (base seca)

$\rho_{1} \ldots \rho_{\mathrm{n}}-$ massa específica dos constituintes de 1 a $\mathrm{n}$, $\mathrm{kg} \mathrm{m}^{-3}$

$\omega_{1} \ldots \omega_{\mathrm{n}}$ - teores dos constituintes do produto de $1 \mathrm{a} \mathrm{n}$, decimal (base seca)

Os teores médios dos constituintes dos grãos de trigo utilizados, segundo a USDA (1975) (Tabela 1) foram:

Tabela 1. Composição química média dos grãos de trigo em $100 \mathrm{~g}$ do produto

\begin{tabular}{lc}
\hline \multicolumn{1}{c}{ Constituinte } & Participação na compocomposição química (mg) \\
Água & $10.270,00$ \\
Proteína & $13.700,00$ \\
Carboidratos (Totais) & $72.570,00$ \\
Vitaminas & 19,60 \\
Lipídios (Totais) & $1.870,00$ \\
Minerais & \\
\hline Cálcio & 34,00 \\
Fósforo & 346,00 \\
\hline Ferro & 3,88 \\
Potássio & 405,00 \\
Cobre & 0,382 \\
Manganês & 3,799 \\
Magnésio & 138,00 \\
Sódio & 5,00 \\
Zinco & 2,93 \\
\hline
\end{tabular}

\section{Forma e tamanho dos grãos}

A forma dos grãos de trigo, considerados esferóides oblatos, foi analisada pela esfericidade (E) e circularidade (C), a partir das medidas das dimensões características, eixos ortogonais (Figura 1) obtidas por paquímetro digital e se considerando as seguintes equações (Mohsenin, 1986):

$$
\begin{gathered}
E=\left[\frac{(a b c)^{1 / 3}}{a}\right] 100 \\
C=\left(\frac{b}{a}\right) 100
\end{gathered}
$$

em que:

$$
\begin{aligned}
& \mathrm{a} \text { - maior eixo do grão, } \mathrm{mm} \\
& \mathrm{b} \text { - eixo médio do grão, } \mathrm{mm} \\
& \mathrm{c}-\text { menor eixo do grão, } \mathrm{mm}
\end{aligned}
$$

b

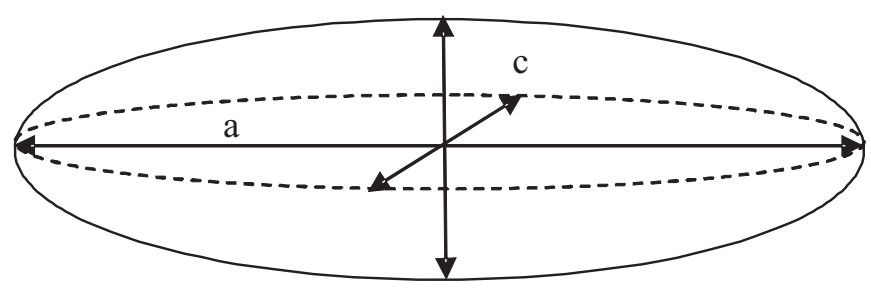

Figura 1. Desenho esquemático do grão de trigo, como esferóide oblato, com suas dimensões características

\section{Contração volumétrica unitária e da massa de grãos}

A contração volumétrica unitária e da massa de grãos durante a secagem do produto, foi definida pela relação entre o volume para cada teor de água e o volume inicial.

Para o acompanhamento da redução do volume da massa, utilizou-se um cilindro de acrílico graduado, enquanto o volume (V) de cada grão foi obtido por meio da medição dos três eixos ortogonais (comprimento, largura e espessura), como proposto por Mohsenin (1986), em vinte grãos, ao longo do processo de secagem e com auxílio de um paquímetro digital, de acordo com a expressão:

$$
\mathrm{V}=\frac{\pi \mathrm{abc}}{6}
$$

\section{Análise estatística}

Os dados experimentais da porosidade, da massa específica real e da massa específica aparente, foram submetidos a análise de regressão, selecionando-se o modelo matemático mais adequado para expressar a relação entre essas características e o teor de água do trigo.

Aos dados experimentais de contração volumétrica unitária e contração volumétrica da massa de grãos, ajustaramse os modelos matemáticos linear, Bala \& Woods (1984) e Rahman (1995), equações 6, 7 e 8, respectivamente; para o ajuste dos modelos aos dados experimentais, foi utilizado o programa computacional Statistica $5.0^{\circledR}$.

$$
\begin{gathered}
\psi_{\mathrm{m}}=\mathrm{A}+\mathrm{BU}^{*} \\
\psi_{\mathrm{m}}=\mathrm{A} \cdot\left\{1-\exp \left[\mathrm{B} \cdot\left(\mathrm{U}^{*}\right)\right]\right\} \\
\psi_{\mathrm{m}}=1+\beta \cdot\left(\mathrm{U}^{*}-\mathrm{U}_{\mathrm{o}}{ }^{*}\right)
\end{gathered}
$$


em que:

$\psi_{\mathrm{m}}$ - índice de contração volumétrica da massa granular, decimal

$\mathrm{U}_{\mathrm{o}}{ }^{*}$ - teor de água inicial do produto, decimal (b.s.)

$\beta$ - coeficiente de contração volumétrica

A, B - parâmetros que dependem do produto

Compararam-se os dados experimentais com os valores calculados pelos modelos, por meio dos erros médio relativo (P) e estimado (SE), conforme descrito a seguir:

$$
\begin{gathered}
\mathrm{P}=\frac{100}{\mathrm{n}} \cdot \sum\left(\frac{|\mathrm{Y}-\hat{\mathrm{Y}}|}{\mathrm{Y}}\right) \\
\mathrm{SE}=\sqrt{\frac{\sum(\mathrm{Y}-\hat{\mathrm{Y}})^{2}}{\mathrm{GLR}}}
\end{gathered}
$$

em que:

$\mathrm{n}$ - número de observações experimentais

$\mathrm{Y}$ - valor observado experimentalmente

$\hat{\mathrm{Y}}$ - valor calculado pelo modelo

GLR - graus de liberdade do modelo (número de dados menos o número de parâmetros do modelo)

Para análise do grau de ajuste de cada modelo foram consideradas as magnitudes do coeficiente de determinação ajustado (variância explicada), do erro médio relativo e estimado e a verificação do comportamento da distribuição dos resíduos.

\section{RESULTADOS E DISCUSSÃO}

Os valores experimentais e estimados da porosidade da massa de grãos estão apresentados na Figura 2.

Observa-se um aumento linear da porosidade na faixa de umidade entre 0,15 e 0,31 (b.s.), concordando com Afonso Júnior et al. (2000) que notaram o mesmo comportamento durante a secagem de grãos de milheto. Para o teor de água 0,12 (b.s.) verifica-se aumento da porosidade devido, provavelmente, a erro experimental; para o armazenamento seguro do trigo, a secagem é interrompida quando o produto apre-

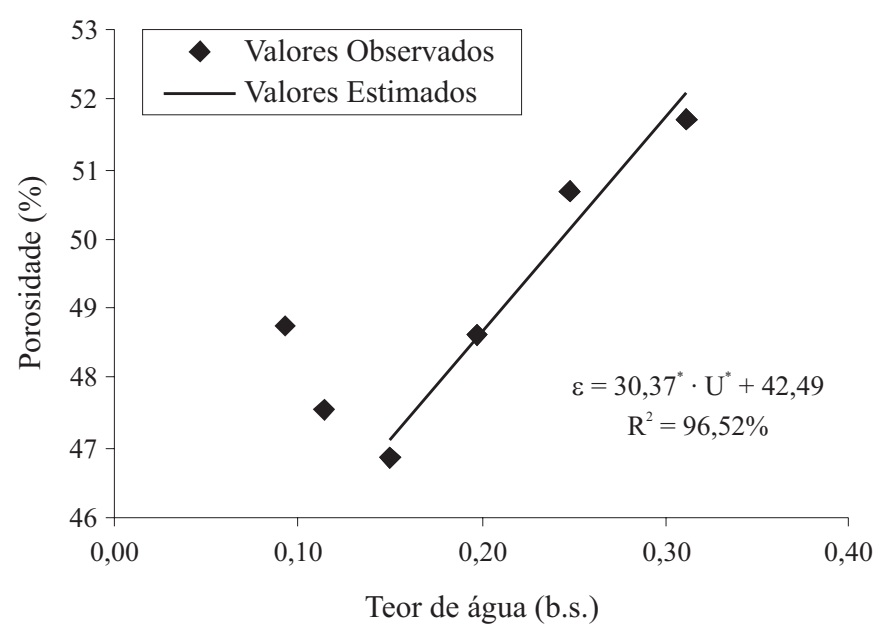

Figura 2. Valores observados e estimados da porosidade $(\varepsilon)$ dos grãos de trigo em função do teor de água $\left(U^{*}\right)$ senta umidade em torno de 0,15 (b.s.); assim, para obtenção do modelo de regressão foram utilizados os valores de porosidade referentes aos teores de água superiores a este valor.

A Figura 3 apresenta os valores experimentais e estimados da massa específica aparente e real e os valores calculados em função da composição química, para diferentes teores de água.
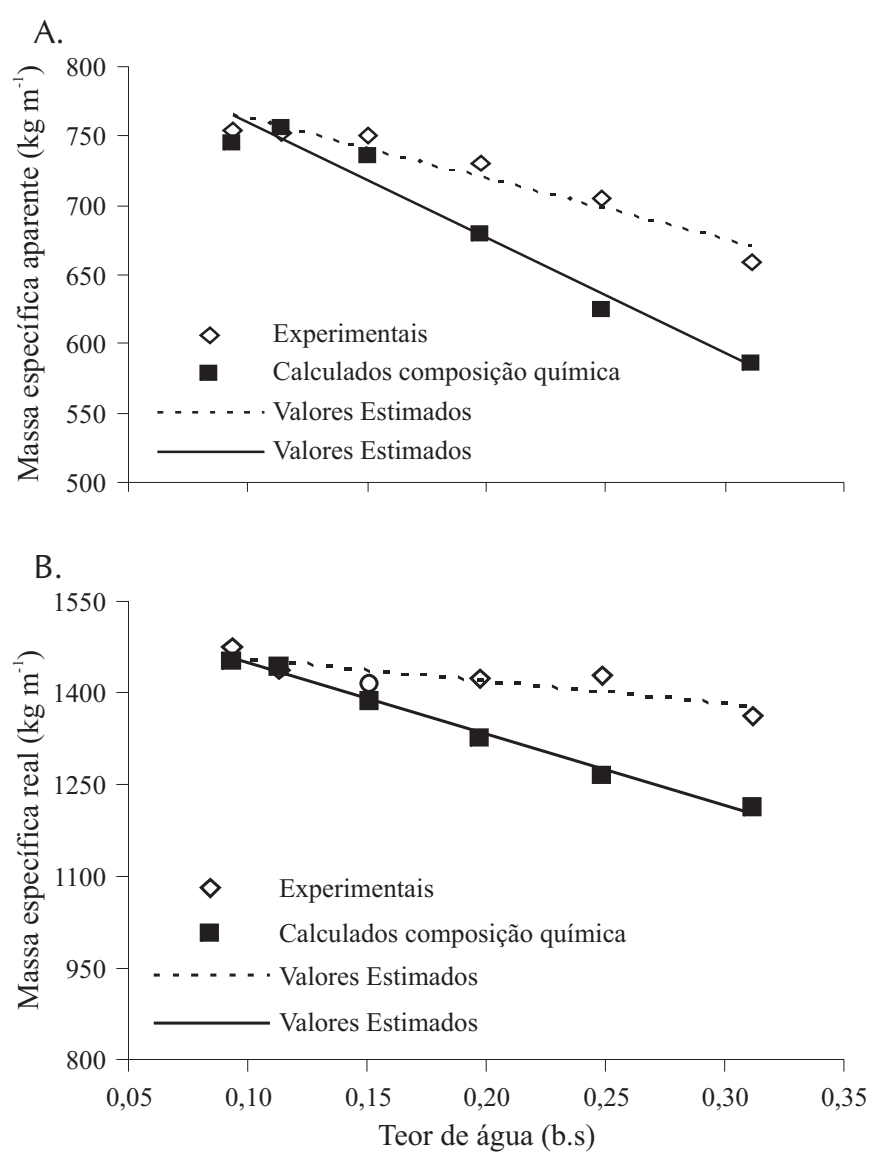

Figura 3. Valores experimentais da massa específica aparente e calculados em função da composição química (A); valores da massa específica real, em função da porosidade e massa específica aparente e calculados pela composição química dos grãos de trigo, para diferentes teores de água (B)

Verifica-se redução da massa específica aparente e da massa específica real com o aumento do teor de água, como observado para a maioria dos produtos agrícolas, independentemente da metodologia utilizada, além de que os valores das massas específicas obtidos em função da composição química do produto, foram inferiores aos dados experimentais; no entanto, constata-se que as diferenças entre os valores experimentais e os calculados diminuíram com a redução do teor de água. Afonso Júnior et al. (2000) calcularam a massa específica real utilizando valores da composição química para grãos de milheto e encontraram resultados superiores aos experimentais, contrário ao verificado para os grãos de trigo, no presente trabalho.

$\mathrm{Na}$ Tabela 2 tem-se os modelos lineares de regressão ajustados aos valores experimentais e calculados de massa específica aparente e massa específica real e os coeficientes de determinação $\left(\mathrm{R}^{2}\right)$ com seus respectivos níveis de significância. 
Tabela 2. Modelos de regressão ajustados aos valores de massa específica aparente e real e os coeficientes de determinação $\left(R^{2}\right)$ com seus respectivos níveis de significância

\begin{tabular}{|c|c|}
\hline Equações de regressão & $R^{2}$ \\
\hline \multicolumn{2}{|l|}{ Massa específica aparente } \\
\hline$\rho_{\mathrm{apl}}=-437,34^{* *} \cdot\left(\mathrm{U}^{*}\right)+806,78^{* *}$ & $0,9272^{++}$ \\
\hline$\rho_{\mathrm{ap}_{2}}=-826,06^{* *} \cdot\left(\mathrm{U}^{*}\right)+841,45^{* *}$ & $0,9610^{++}$ \\
\hline \multicolumn{2}{|l|}{ Massa específica real } \\
\hline$\rho_{\mathrm{r} 1}=-350,14^{\prime \prime} \cdot\left(\mathrm{U}^{*}\right)+1488^{* *}$ & $0,6938^{+}$ \\
\hline$\rho_{\mathrm{r} 2}=-1151,8^{* *} \cdot\left(\mathrm{U}^{*}\right)+1561,3^{* *}$ & $0,9892^{++}$ \\
\hline
\end{tabular}

Analisando os resultados da Tabela 2, vê-se que o modelo linear representa satisfatoriamente, os dados experimentais e calculados da massa específica real e aparente.

Na Tabela 3 encontram-se as equações de regressão para contração volumétrica unitária e da massa de grãos de trigo, em função do teor de água, com seus respectivos coeficientes de determinação, erro médio relativo e estimado, e tendência de distribuição dos resíduos.

Tabela 3. Modelos de contração volumétrica unitária e da massa de grãos de trigo com seus respectivos coeficientes de determinação $\left(R^{2}\right)$, erro médio estimado (SE) e relativo $(\mathrm{P})$, e tendência de distribuição dos resíduos

\begin{tabular}{lcccl}
\hline \multicolumn{1}{c}{ Modelos } & $\mathbf{R}^{2}(\%)$ & $\mathbf{P}$ & SE & $\begin{array}{c}\text { Distribuição } \\
\text { dos Resíduos }\end{array}$ \\
Contração volumétrica da massa & & & & \\
$\Psi_{\mathrm{m}}=1-1,4086\left(\mathrm{U}^{*}-\mathrm{U}_{0}{ }^{*}\right)$ & 86,87 & 3,02423 & 0,00279 Tendenciosa \\
$\Psi_{\mathrm{m}}=1-0,2917 \cdot\left(1-\mathrm{e}^{-9,6598 \cdot\left(\mathrm{U}^{*}-\mathrm{U}_{\mathrm{o}}^{*}\right)}\right)$ & 98,57 & 0,87429 & 0,00032 Aleatória \\
$\Psi_{\mathrm{m}}=0,6049+1,1408 \cdot \mathrm{U}^{*}$ & 94,63 & 1,87059 & 0,00123 Tendenciosa \\
Contração volumétrica unitária & & & & \\
$\Psi_{\mathrm{g}}=1-0,9142\left(\mathrm{U}^{*}-\mathrm{U}_{\mathrm{o}}^{*}\right)$ & 73,34 & 2,52320 & 0,00214 Tendenciosa \\
$\Psi_{\mathrm{g}}=1-0,1642\left(1-\mathrm{e}^{-14,51\left(\mathrm{U}^{*}-\mathrm{U}_{\mathrm{o}}{ }^{*}\right)}\right) 97,96$ & 0,53884 & 0,00017 Aleatória \\
$\Psi_{\mathrm{g}}=0,7531+0,6799 \cdot \mathrm{U}^{*}$ & 89,07 & 1,59245 & 0,00095 Tendenciosa \\
\hline $\mathrm{U}^{*}$ : Teor de água b.s. & & &
\end{tabular}

A análise dos dados indica que o modelo de Bala \& Woods (1984) adaptado foi o que melhor se ajustou aos dados obtidos de contração volumétrica unitária e da massa de grãos de trigo, apresentando tendência menos acentuada de distribuição dos resíduos (distribuição aleatória). Observase, ainda, que este modelo apresentou maior coeficiente de determinação e menores erros médio estimado e relativo. Por outro lado, o modelo linear também representou satisfatoriamente os dados experimentais da contração volumétrica, como observado por Lang \& Sokhansanj (1993) para grãos de trigo e por Lang et al. (1994), Wang \& Brennan (1995) e Afonso Júnior et al. (2000) para diversos produtos agrícolas.

Na Figura 4 são apresentados os valores experimentais da contração volumétrica unitária e da massa de grãos, ajustados pelo modelo de Bala \& Woods (1984) adaptado, em função do teor de água.

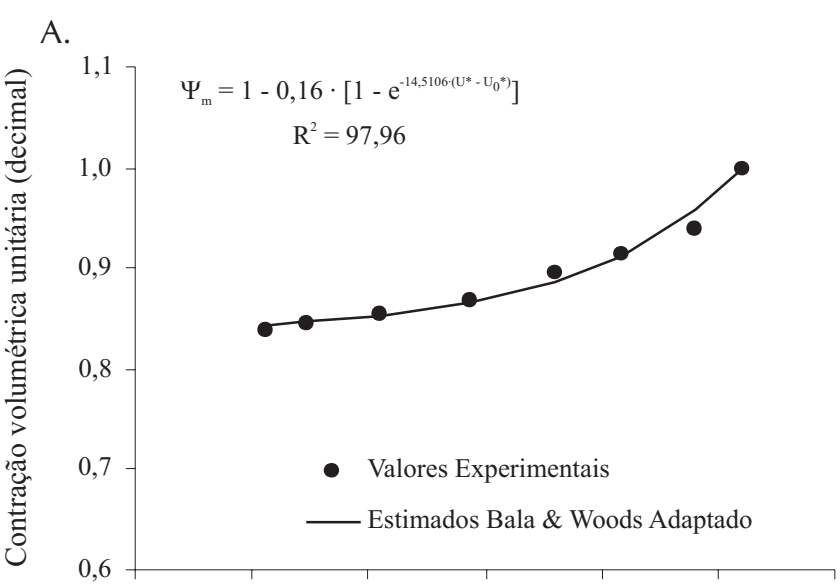

B.

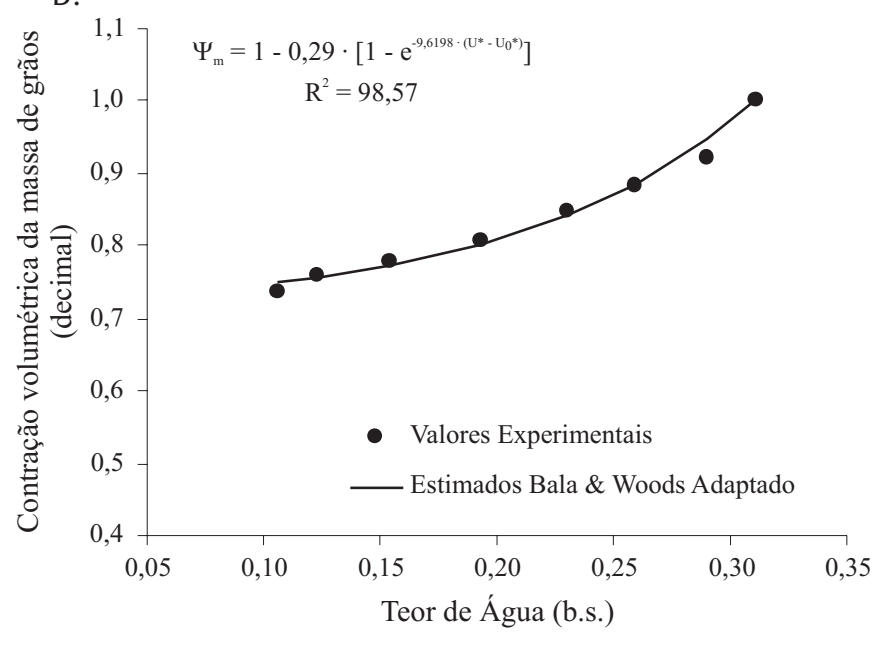

Figura 4. Valores experimentais e estimados pelo modelo de Bala \& Woods adaptado da contração volumétrica unitária (A) e da contração volumétrica da massa de grãos (B) em função do teor de água para o trigo

Verifica-se que a massa de grãos apresentou redução do volume inicial, de $26,33 \%$, e a contração volumétrica unitária, seguindo a mesma tendência, foi de $16,20 \%$ no intervalo de umidade entre 0,31 a 0,11 (b.s.).

$\mathrm{Na}$ Tabela 4 nota-se a variação dos eixos ortogonais dos grãos de trigo, da circularidade e esfericidade em função do teor de água.

Constata-se que as três dimensões dos grãos de trigo reduziram proporcionalmente com a diminuição do teor

Tabela 4. Dimensões médias dos eixos (a, b e c) ortogonais dos grãos de trigo e respectivos índices de redução, esfericidade (E) e circularidade (C)

\begin{tabular}{|c|c|c|c|c|c|c|c|c|}
\hline \multirow{2}{*}{$\begin{array}{l}\text { Teor de } \\
\text { água } \\
\text { (b.s.) }\end{array}$} & \multicolumn{3}{|c|}{ Eixos ortogonais (mm) } & \multirow{2}{*}{$\begin{array}{l}a / a_{0} \\
(\%)\end{array}$} & \multirow{2}{*}{$\begin{array}{l}\mathrm{b} / \mathrm{b}_{0} \\
(\%)\end{array}$} & \multirow{2}{*}{$\begin{array}{l}c / c_{0} \\
(\%)\end{array}$} & \multirow{2}{*}{$\underset{(\%)}{C}$} & \multirow{2}{*}{$\begin{array}{c}E \\
(\%)\end{array}$} \\
\hline & a & b & c & & & & & \\
\hline 0,311 & 6,76 & 3,44 & 3,10 & 100,00 & 100,00 & 100,00 & 50,94 & 61,58 \\
\hline 0,290 & 6,57 & 3,40 & 3,03 & 97,28 & 98,68 & 97,28 & 51,68 & 62,00 \\
\hline 0,259 & 6,49 & 3,37 & 3,01 & 96,13 & 97,81 & 96,13 & 51,83 & 62,17 \\
\hline 0,230 & 6,45 & 3,34 & 2,99 & 95,52 & 97,01 & 95,52 & 51,74 & 62,13 \\
\hline 0,193 & 6,40 & 3,30 & 2,96 & 94,77 & 95,74 & 94,77 & 51,47 & 62,00 \\
\hline 0,154 & 6,38 & 3,28 & 2,95 & 94,37 & 95,28 & 94,37 & 51,43 & 61,93 \\
\hline 0,123 & 6,36 & 3,26 & 2,93 & 94,21 & 94,78 & 94,21 & 51,25 & 61,82 \\
\hline 0,106 & 6,35 & 3,26 & 2,92 & 93,95 & 94,60 & 93,95 & 51,29 & 61,79 \\
\hline
\end{tabular}


de água, opondo-se à maioria dos produtos biológicos porosos que, durante a secagem, se contraem irregularmente nas direções longitudinal, tangencial e radial (Fortes \& Okos, 1980); assim, os valores de esfericidade e circularidade não apresentaram diferenças significativas a nível de $5 \%$ de probabilidade para os teores de água estudados, fato que indica que os grãos de trigo, para a faixa de umidade estudada, apresentam contração uniforme, de acordo com seus eixos principais, resultando na invariabilidade de sua forma durante o processo de dessorção.

\section{CONCLUSÕES}

1. A redução do teor de água influencia as propriedades físicas dos grãos de trigo, provocando diminuição da porosidade e aumento da massa específica aparente e da massa específica real.

2. O modelo de Bala \& Woods adaptado, é o que melhor se ajusta aos dados experimentais de contração volumétrica do grão e da contração volumétrica da massa de grãos de trigo.

3. Os valores das massas específicas real e aparente, calculados a partir da composição química dos grãos de trigo, são inferiores aos valores experimentais. As diferenças entre os valores obtidos pelas duas metodologias, diminuem com a redução do teor de água do produto.

\section{LITERATURA CITADA}

Afonso Júnior, P. C.; Corrêa, P. C.; Andrade, E. T. Análise da variação das propriedades físicas e contração volumétrica dos grãos de milheto (Pennisetum glaucum) durante o processo de dessorção. Revista Brasileira de Armazenamento, Viçosa, v.25, n.1, p.15-21, 2000.

Bala, B. K.; Woods, J. L. Simulation of deep bed malt drying. Journal Agricultural Engineering Research, New York, v.30, n.3, p.235-244, 1984.

Brasil. Ministério da Agricultura e Reforma Agrária. Regra para análise de sementes. Brasília: Mara, 1992. 365p.

Brooker, D. B.; Bakker-Arkema, F. W.; Hall, C. W. Drying and storage of grains and oilseeds. Westport: The AVI Publishing Company, 1992. 450p.

Fortes, M.; Okos, M. R. Changes physical properties of corn during drying. Transaction of ASAE, St. Joseph, v.23, n.4, p.1004-1008, 1980.
Hall, C. W. Drying and storage of agricultural crops. Westport: AVI, 1980. 381p.

Krokida, M. K.; Maroulis, Z. B. Effect of drying method on shrinkage and porosity. Drying Technology, New York, v.15, n.10, p.2441-2458, 1997.

Lang, W.; Sokhansanj, S. Bulk volume shrinkage during drying of wheat and canola. Journal of Food Process Engineering, Trumbull, v.16, n.4, p.305-314, 1993.

Lang, W.; Sokhansanj, S.; Rohani, S. Dynamic shrinkage and variable parameters in Bakker-Arkema's mathematical simulation of wheat and canola drying. Drying Technology, New York, v.12, n.7, p.1687-1708, 1994.

Lewis, M. J. Propriedades físicas de los alimentos y de los sistemas de procesados. Zaragoza: Editorial Acribia S.A., 1993. 494p.

Mayor, L.; Sereno, A. M. Modelling shrinkage during convective drying of food materials: a review. Journal of Food Engineering, London, v.61, p.373-386, 2004.

Mcminn, W. A. M.; Magee, T. R. A. Physical characteristics of dehydrated potatoes - part I. Journal of Food Engineering, London, v.33, n.1-2, p.37-48, 1997.

Mohsenin, N. N. Physical properties of plant and animal materials. New York: Gordon and Breach Publishers, 1986. 841p.

Rahman, S. Physical properties of foods. Boca Raton, Florida: CRC Press LLC, 1995. 500p.

Ratti, C. Shrinkage during drying of foodstuffs. Journal of Food Engineering, London, v.23, n.1, p.91-105, 1994.

Ruffato, S.; Corrêa, P. C.; Martins, J. H.; Mantovani, B. H. M.; Silva, J. N. Influência do processo de secagem sobre a massa específica aparente, massa específica unitária e porosidade de milho-pipoca. Revista Brasileira de Engenharia Agrícola e Ambiental, Campina Grande, v.3, n.1, p.45-48, 1999.

Sokhansanj, S.; Lang, W. Prediction of kernel and bulk volume of wheat and canola during adsorpsion and desorption. Journal Agricultural Engineering Research, New York, v.63, n.2, p.129-136, 1996.

Towner, G. D. The tensile stress generated in clay through drying. Journal Agricultural Engineering Research, New York, v.37, n.4, p.279-289, 1987.

USDA. United States Department of Agriculture. Handbook of the nutritional contents of foods. New York: Dover Publications Inc., 1975. 190p.

Wang, N.; Brennan, J. G. Changes in structure, density and porosity of potato during dehydration. Journal of Food Engineering, London, v.24, n.1, p.61-76, 1995.

Zogzas, N. P.; Maroulis, Z. B.; Kouris, D. M. Densities, shrinkage and porosity of some vegetables during air drying. Drying Technology, New York, v.12, n.7, p.1653-1666, 1994. 